\section{ORIGINAL RESEARCH}

D.W. Kim

C.K. Eun

H.S. In

M.H. Kim

S.J. Jung

S.K. Bae

\title{
Sonographic Differentiation of Asymptomatic Diffuse Thyroid Disease from Normal Thyroid: A Prospective Study
}

BACKGROUND AND PURPOSE: There is no useful guide or study related to the differentiation of asymptomatic diffuse thyroid disease from normal thyroid by using thyroid US. This study was prospectively designed to evaluate the efficacy of the use of real-time thyroid sonography as performed by an experienced radiologist for the identification of asymptomatic DTD.

MATERIALS AND METHODS: From January 2008 to December 2008, 2267 patients underwent thyroid sonography in our hospital by 1 radiologist. Each patient's thyroid was prospectively classified as being in 1 of 4 of the following diagnostic categories on the basis of the sonographic features as determined with the use of real-time sonography: suggestive for DTD, suspicious for DTD, indeterminate, and no evidence of DTD. We calculated the diagnostic efficacy of the sonographic classifications compared with the pathology results.

RESULTS: Sonographic classifications for DTD in 340 patients who underwent thyroid surgery because of thyroid malignancy or other causes included the following: suggestive for DTD $(n=32)$, suspicious for DTD $(n=39)$, indeterminate $(n=18)$, and no evidence of DTD $(n=251)$. On the pathology, HT $(n=$ $33)$, chronic lymphocytic thyroiditis $(n=27)$, diffuse hyperplasia $(n=2)$, and NTP $(n=278)$ were identified. There were true-positive cases $(n=50)$, true-negative cases $(n=244)$, false-positive cases $(n=21)$, and false-negative cases $(n=7)$. The sensitivity, specificity, positive predictive value, negative predictive value, and accuracy for a diagnosis of asymptomatic DTD were $87.7 \%, 92.1 \%$, $70.4 \%, 97.2 \%$ and $91.3 \%$, respectively.

CONCLUSIONS: The present sonographic classification based on real-time sonography of the thyroid is a useful tool for differentiating asymptomatic DTD from normal thyroid.

\begin{abstract}
ABBREVIATIONS: $\mathrm{AP}=$ anteroposterior; $\mathrm{CLT}=$ chronic lymphocytic thyroiditis; $\mathrm{DH}=$ diffuse hyperplasia; DTD = diffuse thyroid disease; $\mathrm{HT}=$ Hashimoto thyroiditis; NPV = negative predictive value; NTP = normal thyroid parenchyma; PPV = positive predictive value; PTC = papillary thyroid carcinoma; TPOAb = antithyroperoxidase antibody; US = sonography
\end{abstract}

$\mathbf{T}$ hyroid sonography has been used as the major diagnostic technique for the evaluation of thyroid disease, especially in the evaluation of nodular thyroid disease. Nonetheless, the use of thyroid sonography in the evaluation of DTD has been restricted to the following: the screening of nodular thyroid disease for DTD, the differentiation or characterization of DTD, or the detection of a diffuse infiltrating tumor. ${ }^{1-7}$

For the sonographic evaluation of DTD, several helpful sonographic features have been used, including decreased or increased parenchymal echogenicity, a coarse echotexture, decreased or increased vascularity, a decreased or increased AP diameter of the gland, the presence of marginal nodularity, and the presence of scattered microcalcifications. ${ }^{1-7}$ Ralls et $\mathrm{al}^{1}$ reported the "thyroid inferno" as a useful sonographic feature on color Doppler sonography for the identification of Graves disease. Yeh et $\mathrm{al}^{2}$ introduced "micronodulation" as a specific sonographic sign of HT. However, thyroid sonography has

Received August 12, 2009; accepted after revision April 19, 2010.

From the Departments of Radiology (D.W.K., C.K.E., H.S.I., M.H.K.), Nuclear Medicine (S.K.B.), and Pathology (S.J.J.), Busan Paik Hospital, Inje University College of Medicine, Busan, South Korea.

This work was supported by the Busan Paik Hospital Imaging Research Institute.

Please address correspondence to Dong Wook Kim, MD, Department of Radiology, Busan Paik Hospital, Inje University College of Medicine, Busan, South Korea; e-mail: dwultra@ lycos.co.kr

$\equiv$ indicates article with supplemental on-line tables.

DOI 10.3174/ajnr.A2164 had a limited role in the diagnosis of DTD because clinical and laboratory findings have played a significant role in the diagnosis and treatment of this condition.

Real-time sonography of the thyroid for the differentiation of DTD from normal thyroid is ideal. Nordmeyer et $\mathrm{al}^{3}$ suggested that thyroid sonography was of considerable value in establishing the absence of autoimmune thyroiditis because autoimmune thyroiditis could be excluded in $84 \%$ of cases on the basis of the use of a prospective sonographic examination alone. To the best of our knowledge, however, a prospective sonographic study for the identification of asymptomatic DTD with the use of a real-time thyroid sonography has not been reported.

The aim of this study was to assess the efficacy of a prospective sonographic diagnosis of asymptomatic DTD by an experienced radiologist when correlated with the pathology results after surgery of the thyroid.

\section{Materials and Methods}

\section{Patient Selection}

From January 2008 to December 2008, 1 radiologist (D.W.K.) performed thyroid sonography by using a high-resolution sonography instrument (iU 22 Ultrasound System; Philips Medical Systems, Bothell, Washington) equipped with a $15-\mathrm{MHz}$ linear probe in patients who underwent a first sonographic evaluation of the thyroid in our hospital, regardless of previous thyroid sonography examinations performed in other hospitals. A total of 2267 patients (females/males, 

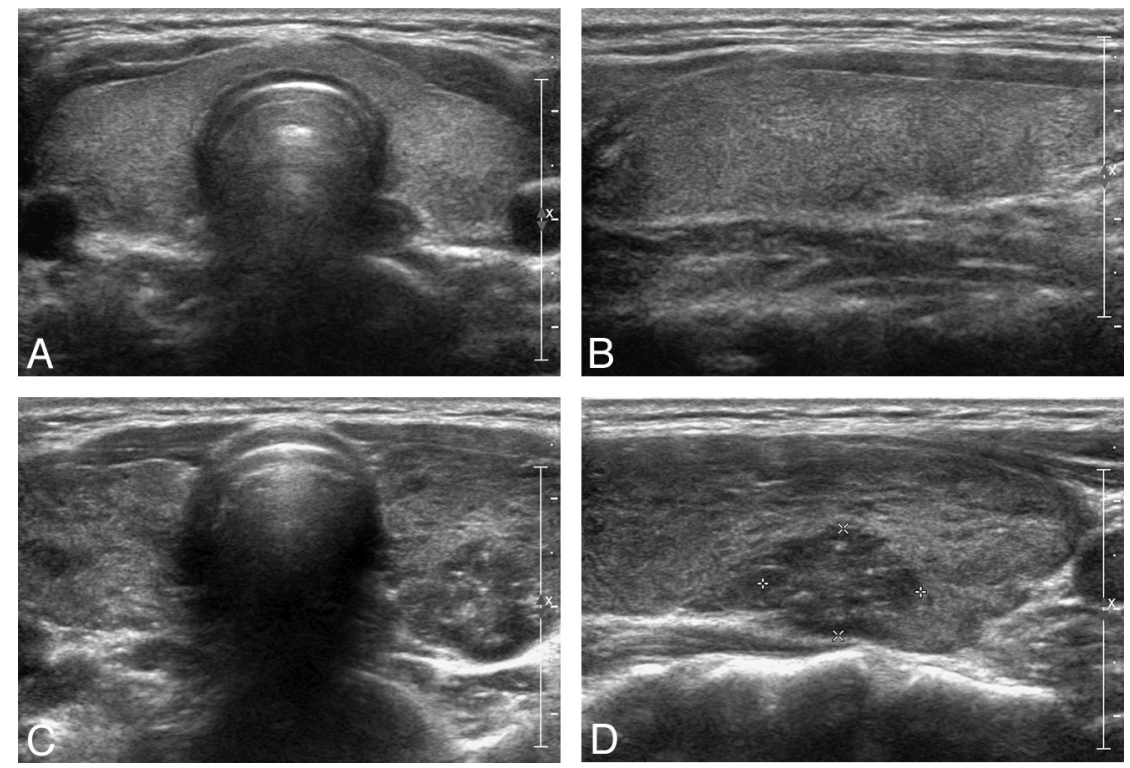

Fig 1. Category of no evidence of DTD in a 28-year-old woman. $A$ and $B$, Transverse and longitudinal sonographic images of the thyroid show isoechogenicity, fine echotexture, an AP diameter from 1 to $2 \mathrm{~cm}$, normal vascularity, a smooth margin, and the absence of scattered microcalcifications. Pathology results (not shown) showed a normal thyroid after thyroid surgery because of a follicular adenoma in the left lobe. $C$ and $D, A$ category of suggestive for DTD of the thyroid in a 33-year-old woman. Transverse and longitudinal sonographic images of the thyroid show mild hypoechogenicity, coarse echogenicity, mildly increased vascularity, and the presence of a microlobulated margin. Pathology results showed HT and a papillary thyroid carcinoma in the left lobe after thyroid surgery.

1898:369; age range, $11-85$ years; mean age, $47.6 \pm 12.3$ years) were enrolled in the study. The institutional review board approved this study.

The inclusion criteria for patients undergoing a prospective sonographic diagnosis for asymptomatic DTD included a first thyroid sonography examination performed in our hospital regardless of any previous thyroid sonography examination performed in another hospital, the use of the same high-resolution sonography instrument, the same operator in the performance of the thyroid sonography examination, and a time interval of $\leq 3$ months from the thyroid sonography examination to thyroid surgery. Patients who met the following criteria were excluded from the study: known DTD, known clinical symptoms or abnormal laboratory findings related to DTD, or a history of previous thyroid surgery. Also, laboratory findings including serum levels of thyroid hormones, thyroid-stimulating hormone, and thyroid autoantibodies were retrospectively assessed; the prospective sonographic diagnosis was designed to be blinded to laboratory information.

\section{Sonographic Classification of the Thyroid}

The sonographic features related to DTD were the following: The sonographic characteristics of echogenicity included isoechoic, hypoechoic, markedly hypoechoic, and hyperechoic patterns. The strap muscles and submandibular glands were used as a reference for the determination of echogenicity. The sonographic characteristics of echotexture included fine, coarse, and micronodulative patterns. The AP diameter of the thyroid on a longitudinal scan was divided into 3 categories: a normal range from 1 to $2 \mathrm{~cm},<1 \mathrm{~cm}$, and $>2 \mathrm{~cm}$. The sonographic categories of glandular vascularity included normal, mildly increased, markedly increased, and decreased patterns. The margin of the thyroid was classified as having smooth, microlobulated, and macrolobulated patterns. The investigator considered patient age in the determination of the degree of sonographic features during a thyroid sonography examination on the basis of individual experience because the sonographic features of the thyroid might change with age.

The thyroid was prospectively classified into 1 of 4 categories on the basis of real-time sonography features, including echogenicity, echotexture, AP diameter, vascularity, a glandular margin, and the presence of scattered microcalcifications (Fig 1). The categories included suggestive for DTD, suspicious for DTD, indeterminate, and no evidence of DTD. The criteria for prospective sonographic diagnosis of asymptomatic DTD included the following: If a thyroid showed $\geq 3$ sonographic characteristics related to DTD as depicted on a real-time thyroid sonography, it was classified as suggestive for DTD. If a thyroid showed 2 sonographic characteristics related to DTD, it was classified as suspicious for DTD. If a thyroid showed only 1 sonographic feature related to DTD, it was classified as indeterminate. If a thyroid showed isoechogenicity, fine echotexture, an AP diameter from 1 to $2 \mathrm{~cm}$, normal vascularity, a smooth margin, and the absence of scattered microcalcifications, it was classified as having no evidence of DTD. All sonographic diagnoses of the thyroid were prospectively determined by 1 radiologist on the basis of real-time sonography.

\section{Determination of Reference Standards and Diagnostic Index}

We retrospectively compared the prospective sonographic diagnoses and the pathologic results. The thyroid abnormalities that were depicted on thyroid sonography as suspicious for DTD and suggestive for DTD were classified as positive, and a sonographic diagnosis of no evidence of DTD was classified as negative. The indeterminate category was excluded in the determination of the diagnostic efficacy of thyroid sonography.

The pathologic criteria of HT included a progressive loss of thyroid follicular cells, a concomitant replacement of the gland by lymphocytes, and the formation of germinal centers associated with fibrosis. We classified a thyroid showing diffuse infiltration of 

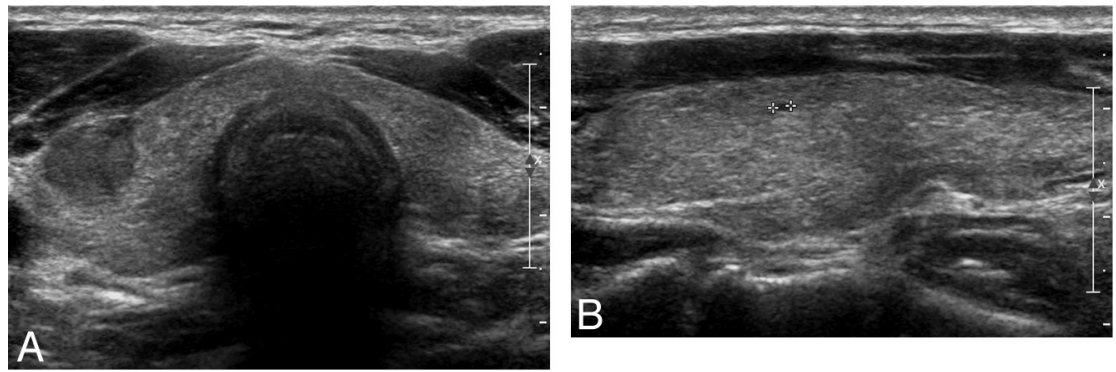

Fig 2. Category of suggestive for DTD in a 39-year-old man (a false-positive). $A$ and $B$, Transverse and longitudinal sonographic images of the thyroid show mild hypoechogenicity, coarse echogenicity, and the presence of a microlobulated margin, but the thyroid pathology results showed a papillary thyroid carcinoma in the left lobe and NTP after thyroid surgery.

lymphocytes and other inflammation-related cells and no evidence of typical pathologic findings of HT, such as oxyphilic metaplasia, follicular atrophy, or follicular disruption, as chronic lymphocytic thyroiditis.

The diagnostic indices (sensitivity, specificity, positive predictive value, negative predictive value, and accuracy) for the use of the present sonographic classifications and individual sonographic features for the identification of asymptomatic DTD were calculated.

Statistical analyses were performed with the use of the Statistical Package for the Social Sciences software, Version 12.0 for Windows (SPSS, Chicago, Illinois). Multiple logistic regression analysis was used to determine independent sonographic criteria for the identification of asymptomatic DTD. A 2-tailed $P$ value $<.05 \%$ was considered statistically significant.

\section{Results}

\section{Sonographic Classifications for the Thyroid}

Of the 2267 patients who had thyroid sonography during the study period, 340 patients (women/men $=295: 45$; age range, from 20 to 75 years; mean age, 46.1 years \pm 10.7 years) underwent thyroid surgery in this hospital. Surgery was performed because of a known thyroid malignancy cytologically diagnosed in another hospital ( $n=182)$, a cytologically diagnosed thyroid malignancy identified in this hospital $(n=126)$, a benign thyroid lesion $(n=31)$, and a parathyroid lesion $(n=$ $1)$. After thyroid surgery, PTC $(n=306)$, follicular thyroid carcinoma $(n=3)$, medullary thyroid carcinoma $(n=1)$, follicular adenoma $(n=4)$, parathyroid adenoma $(n=1)$, and nodular hyperplasia ( $n=25$ ) were identified by pathology. Each thyroid was prospectively classified as the following on the basis of the features of real-time thyroid sonography: suggestive for DTD $(n=32)$, suspicious for DTD $(n=39)$, indeterminate $(n=18)$, and no evidence of DTD $(n=251)$.

\section{Correlation between Thyroid Sonography Classifications and Pathologic Findings}

All 340 patients had a pathologic diagnosis for asymptomatic DTD or a NTP after thyroid surgery. The mean time interval between thyroid sonography and thyroid surgery was 0.94 months (range, 0.1-2.5 months). On the pathologic results, HT $(n=33)$, chronic lymphocytic thyroiditis $(n=27)$, diffuse hyperplasia $(n=2)$, and the NTP $(n=278)$ were identified. On the basis of laboratory data for the patients having chronic thyroiditis on the pathology, 14 patients were confirmed as having HT (14/60, 23.3\%) because of positive TPOAb levels in serum, but other patients having chronic thyroiditis on pa- thology were confirmed as having focal lymphocytic thyroiditis $(46 / 60,76.7 \%)$ because of a normal range of TPOAb levels in serum. The incidence of sonographic features of the thyroid in 322 patients is shown on the basis of the pathologic results when 18 cases of indeterminate category were excluded (Online Table 1).

A comparison of the sonographic diagnoses and the pathologic results for 340 patient thyroids is analyzed into the following: The 32 cases assigned to the category of suggestive of DTD by thyroid sonography included HT $(n=22)$, chronic lymphocytic thyroiditis $(n=5)$, diffuse hyperplasia $(n=1)$, and NTP $(n=4)$. The 39 cases assigned to the category of suspicious for DTD included HT $(n=7)$, chronic lymphocytic thyroiditis $(n=14)$, diffuse hyperplasia $(n=1)$, and NTP $(n=17)$ (Fig 2$)$. The 18 cases assigned to the indeterminate category included HT $(n=3)$, chronic lymphocytic thyroiditis $(n=2)$, and NTP $(n=13)$. The 251 cases assigned to the category of no evidence of DTD included HT $(n=1)$, chronic lymphocytic thyroiditis $(n=6)$, and NTP $(n=244)$ (Fig 3). In the frequency analysis of the sonographic features for asymptomatic DTD and NTP, isoechogenicity, fine echotexture, an AP diameter from 1 to $2 \mathrm{~cm}$, normal vascularity, and the presence of a smooth margin were shown to have a significant association for the identification of NTP $(P<.01)$, but marked hypoechogenicity, micronodulation, an AP diameter $>2 \mathrm{~cm}$, markedly increased vascularity, and the presence of a macrolobulated margin were significantly associated with asymptomatic DTD $(P<.01)$.

\section{Diagnostic Index of Thyroid Sonography}

When the thyroids $(n=18)$ assigned to the indeterminate category were excluded, there were 50 true-positive cases, 21 false-positive cases, 244 true-negatives cases, and 7 false-negative cases for diagnoses. The diagnostic indices of individual sonography features for asymptomatic DTD are described in On-line Table 2. The sensitivity, specificity, positive predictive value, negative predictive value, and accuracy of the present sonographic classification system for the identification of asymptomatic DTD were $87.7 \%, 92.1 \%, 70.4 \%, 97.2 \%$ and $91.3 \%$, respectively. The diagnostic indices of the present sonographic classification for the identification of asymptomatic DTD showed a high value except for the positive predictive value. Furthermore, multiple logistic regression analysis was performed to determine the significance of independent sonographic features for the identification of asymptomatic DTD. For the identification of asymptomatic DTD on thyroid sonography, coarse echotexture, micronodulation, mildly in- 


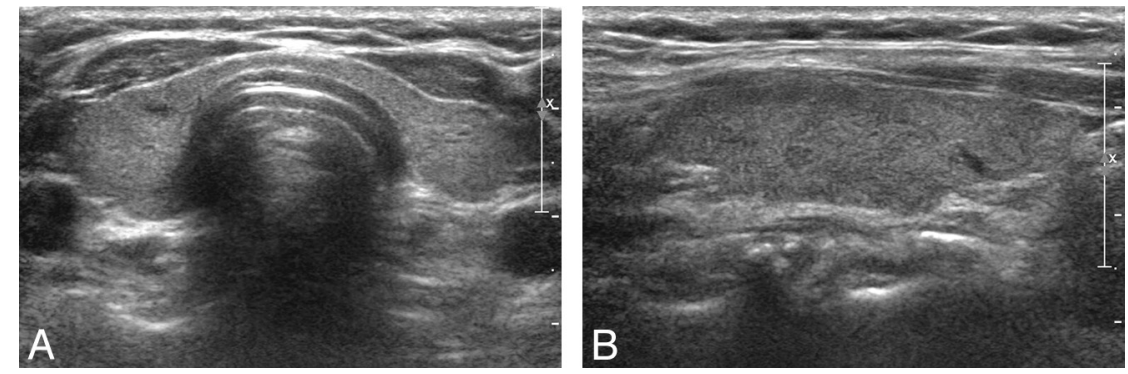

Fig 3. Category of no evidence of DTD in a 59-year-old woman (a false-negative). $A$ and $B$, Transverse and longitudinal sonographic images of the thyroid show fine isoechogenicity, a normal range for the AP diameter, and the presence of a smooth margin. Pathology results showed a papillary thyroid carcinoma in the left lobe and chronic lymphocytic thyroiditis after thyroid surgery.

creased vascularity, and the presence of a macrolobulated margin showed a statistically significant association $(P<.05)$.

\section{Discussion}

High-resolution thyroid sonography is a useful diagnostic tool for the evaluation of DTD. There are numerous published studies related to the diagnostic efficacy of thyroid sonography for DTD, and several sonographic characteristics have been described as potential predictors of DTD, such as thyroid inferno in Graves disease and micronodulation in HT. ${ }^{1,2} \mathrm{Be}-$ cause nearly all studies related to the sonographic characteristics of DTD have been retrospectively performed, retrospective study might have a significant limitation for the accurate assessment of sonography features of the thyroid. ${ }^{1-7}$

The present study attempted to determine the diagnostic efficacy of the use of real-time sonography for asymptomatic DTD. The sonographic diagnoses of the thyroid parenchyma were classified into 4 categories. Most cases assigned to the suggestive for DTD category as determined on thyroid sonography were confirmed as HT, chronic lymphocytic thyroiditis, and diffuse hyperplasia after thyroid surgery. Only 4 cases that were classified in the category of suggestive for DTD showed NTP on pathology. Therefore, the thyroid has a high likelihood of having asymptomatic DTD if $\geq 3$ abnormal sonographic features, including echogenicity, echotexture, vascularity, AP diameter, and glandular margin, are identified on real-time sonography. For the category of suspicious for DTD, the incidence of DTD was not high compared with the incidence of the NTP. For the category of indeterminate, the incidence of DTD was low compared with that of NTP. In addition, most cases of asymptomatic DTD that were classified in the category of suggestive for DTD were confirmed as HT or chronic lymphocytic thyroiditis. Most cases that were classified in the category of no evidence of DTD showed the NTP on the basis of the pathologic findings after thyroid surgery.

Therefore, we believe that a combination of $\geq 3$ sonographic features of DTD has a high sensitivity and specificity for the identification of DTD compared with the use of $\leq 2$ sonographic features of DTD. We are also convinced that no visualization of sonographic features related to DTD on realtime thyroid sonography can rule out the existence of asymptomatic DTD. Ultimately, the present sonographic classification system showed a high efficacy and accuracy for the identification of asymptomatic DTD, except for a low positive predictive value. However, this study did not assess the combinations of sonographic features for asymptomatic DTD that were more sensitive, specific, or accurate.
There was no individual sonographic feature that showed a high sensitivity and specificity for the identification of asymptomatic DTD in the present study. Yeh et $\mathrm{al}^{2}$ suggested that micronodulation is a specific sonographic sign of HT. In this study, micronodulation had high specificity but low sensitivity because of low incidence. In addition, coarse echogenicity, mildly increased vascularity, and the presence of a microlobulated margin showed high specificity but low sensitivity for the detection of asymptomatic DTD. However, several sonographic features, including marked hypoechogenicity, an AP diameter $>2 \mathrm{~cm}$, markedly increased vascularity, and the presence of a macrolobulated margin, showed very high specificity and very low sensitivity. Gutekunst et $\mathrm{al}^{8}$ reported that $5.4 \%$ of cases of HT appeared as normal findings on thyroid sonography. In this study, $11.3 \%$ (7/62) of the cases of asymptomatic DTD did not demonstrate abnormal sonographic features on real-time thyroid sonography. Therefore, asymptomatic DTD may be mistaken for the normal thyroid, though a high-resolution sonography instrument was used and an experienced radiologist performed the thyroid sonography.

In the present study, there was a high incidence of asymptomatic DTD $(18.2 \%, 62 / 340)$. This result does not represent the general incidence of asymptomatic DTD in South Korea. Asymptomatic DTD, especially HT, is known to be significantly related to the occurrence of thyroid malignancy compared with normal thyroid. ${ }^{9,10}$ Ohmori et $\mathrm{al}^{11}$ reported that PTC with HT has a prevalence of 5.5\%, which is higher than that of PTC without HT. Therefore, we suggest that the high incidence of asymptomatic DTD reflects thyroid malignancy in 310 patients $(91.2 \%, 310 / 340)$ as the main cause of thyroid surgery.

In our study, a relatively low number of patients having asymptomatic autoimmune thyroiditis, including chronic lymphocytic thyroiditis or HT, showed positive TPOAb levels on serologic results $(21 / 60,35 \%)$. However, several studies have reported a high accuracy of the TPOAb with regard to the presence of subclinical hypothyroidism. ${ }^{12,13}$ We think that further study is necessary for the evaluation of the interrelationship between asymptomatic autoimmune thyroiditis and laboratory data, including autoantibodies.

There was no case of a diffuse infiltrating malignancy in this study. The early detection of a diffuse infiltrating tumor in the thyroid is important for the improvement of patient prognosis. Some investigators have suggested that scattered microcalcifications and heterogeneous echotexture could be helpful sonographic features for the identification of the diffuse sclerosing variant of PTC. ${ }^{14}$ However, there was no case showing 
scattered microcalcifications on a real-time thyroid sonography in this study. Hoang et $\mathrm{al}^{15}$ reported that sonographic features suggestive of diffuse infiltrating thyroid malignancy include irregular or nodular enlargement of the thyroid gland, sparing from the infiltrative process in portions of the gland, and the presence of nodal metastases.

There are several limitations to this study. First, most of the study patients underwent thyroid surgery for the treatment of a thyroid malignancy, which can explain the high incidence of autoimmune thyroiditis. Various cases of DTD, including non-autoimmune thyroiditis, a diffuse infiltrating malignancy, or other types of DTD, were not included. Second, patients younger than 20 years of age were not included because their thyroids were not surgically confirmed. Third, we excluded 18 cases of the indeterminate category from the determination of the diagnostic index of thyroid sonography for the identification of DTD. This exclusion might have created a statistical bias. Sonographic features of the 18 cases included coarse echotexture $(n=7)$, AP diameter from 1 to $2 \mathrm{~cm}(n=4)$, mildly increased vascularity $(n=5)$, and the presence of a microlobulated margin $(n=2)$. However, there was no case having thyroid inferno or micronodulation in the indeterminate category. Finally, the AP diameter of the thyroid was used for the assessment of glandular size because of easy acquisition. However, the accuracy of the use of the AP diameter for the determination of thyroid volume is not high. For accurate calculation of thyroid volume, several methods have been introduced for its quantification. Some investigators have suggested that $3 \mathrm{D}$ sonography for thyroid volumetry is useful, that volumetric sonography is better than B-mode sonography on the basis of the reference of pathologic anatomy, and that $3 \mathrm{D}$ volumetric quantification of the thyroid is possible with the use of semiautomated volumetric sonography. ${ }^{16,17}$

In summary, the present sonographic classification based on real-time sonography of the thyroid is a useful tool to differentiate asymptomatic DTD from normal thyroid.

\section{References}

1. Ralls PW, Mayekawa DS, Lee KP, et al. Color-flow Doppler sonography in Graves disease: "thyroid inferno." AJR Am J Roentgenol 1988;150:781-84

2. Yeh HC, Futterweit W, Gilbert P. Micronodulation: ultrasonographic sign of Hashimoto's thyroiditis. J Ultrasound Med 1996;15:813-19

3. Nordmeyer JP, Shafeh TA, Heckmann C. Thyroid sonography in autoimmune thyroiditis: a prospective study on 123 patients. Acta Endocrinol (Copenh) 1990;122:391-95

4. Schiemann U, Avenhaus W, Konturek JW, et al. Relationship of clinical features and laboratory parameters to thyroid echogenicity measured by standardized grey scale ultrasonography in patients with Hashimoto's thyroiditis. Med Sci Monit 2003;9:13-17

5. Pedersen OM, Aardal NP, Larssen TB, et al. The value of ultrasonography in predicting autoimmune thyroid disease. Thyroid 2000;10:251-159

6. Marcocci C, Vitti P, Cetani F, et al. A thyroid ultrasonography helps to identify patients with diffuse lymphocytic thyroiditis who are prone to develop hypothyroidism. J Clin Endocrinol Metab 1991;72:209-13

7. Lai SM, Chang TC, Chang CC, et al. Sonographic presentation in autoimmune thyroiditis. J Formos Med Assoc 1990;89:1057-62

8. Gutekunst R, Hafermann W, Mansky T, et al. Ultrasonography related to clinical and laboratory findings in lymphocytic thyroiditis. Acta Endocrinol (Copenh) 1989;121:129-35

9. Dailey ME, Lindsay S, Skahen R. Relation of thyroid neoplasms to Hashimoto disease of the thyroid gland. AMA Arch Surg 1955;70:291-97

10. Matsubayashi S, Kawai K, Matsumoto Y, et al. The correlation between papillary thyroid carcinoma and lymphocytic infiltration in the thyroid gland. J Clin Endocrinol Metab 1995;80:3421-24

11. Ohmori N, Miyakawa M, Ohmori K, et al. Ultrasonographic findings of papillary thyroid carcinoma with Hashimoto's thyroiditis. Intern Med 2007;46:547-50

12. Rosario PW, Bessa B, Valadao MM, et al. Natural history of mild subclinical hypothyroidism: prognostic value of ultrasound. Thyroid 2009;19:9-12

13. Raber W, Gessl A, Nowotny P, et al. Thyroid ultrasound versus antithyroid peroxidase antibody determinations: a cohort study of four hundred fifty-one subjects. Thyroid 2002;12:725-31

14. Lee JY, Shin JH, Han BK, et al. Diffuse sclerosing variant of papillary carcinoma of the thyroid: imaging and cytologic findings. Thyroid 2007;17:567-73

15. Hoang JK, Lee WK, Lee M, et al. US features of thyroid malignancy: pearls and pitfalls. Radiographics 2007;27:847-65

16. Schlogl S, Werner E, Lassmann M, et al. The use of three-dimensional ultrasound for thyroid volumetry. Thyroid 2001;11:569-74

17. Malago R, D'Onofrio $M$, Ferdeghini $M$, et al. Thyroid volumetric quantification: comparative evaluation between conventional and volumetric ultrasonography. J Ultrasound Med 2008;27:1727-33 